\title{
Modello di tenuta della flangia bullonata, senza guarnizione, mediante l'analogia della meccanica della frattura di una fessura parzialmente aperta
}

\author{
M. Beghini, L. Bertini, C. Santus \\ Università di Pisa, Dipartimento di Ingegneria Meccanica, Nucleare e della Produzione. Largo Lucio Lazzarino 1, 56126 Pisa. \\ ciro.santus@ing.unipi.it \\ C. Cagnarini, P. Romanello \\ General Electric, Oil \& Gas, Nuovo Pignone - 50127 Firenze.
}

RIASsUNTO. I compressori centrifughi di elevate dimensioni non permettono l'utilizzo di guarnizioni deformabili, per cui le due metà della flangia di connessione sono forzate mediante bullonatura e la tenuta è affidata al contatto completo delle due superfici. La previsione della pressione di perdita è un aspetto di progetto di notevole interesse per questa tecnologia. L'azione della pressione interna sollecita la separazione delle superfici della flangia, che invece è contrastata dall'azione di serraggio dei bulloni. Il presente lavoro propone un modello per prevedere la condizione di perdita, basato sulla meccanica della frattura. Dato che le due superfici della flangia sono semplicemente a contatto, esse costituiscono una vera e propria fessura parzialmente aperta. Come ben noto il fattore di intensificazione di una fessura parzialmente aperta è nullo. Imponendo che le due superfici siano parzialmente separate ad una distanza fino al bordo del foro del bullone (che offre un canale di fuoriuscita per il fluido in pressione), e imponendo la condizione di fattore di intensificazione nullo, è possibile determinare la pressione di perdita, analiticamente, mediante la tecnica delle "weight functions" (o "funzioni peso"). Il presente lavoro riporta una positiva validazione del modello proposto mediante sia simulazione numerica sia risultati sperimentali in piena scala e in scala ridotta. Il modello analitico proposto offre uno strumento di progetto di immediata implementazione per comparare diverse geometrie di flangia bullonata.

ABSTRACT. The use of a gasket made in soft material is not recommended for large size centrifugal compressor case flanges. The two case halves are assembled with bolted flanges and the leakage is prevented by the "metalto-metal" contact of the flange surfaces. The prediction of the leakage condition is an important engineering challenge for this technology. A new model to predict the leakage condition, based on Fracture Mechanics, is here presented. The partially open flange surfaces interface can be regarded as a partially open crack. The Stress Intensity Factor of a partially open crack is zero, since the flange surfaces can not transfer tensile traction, being just in contact (not "glue" or "welded"). The extension of the open zone, i.e. the crack length, can be obtained imposing the zero Stress Intensity Factor condition. The leakage is expected as the flange surface open front reaches the bolt hole, that produces a way out path for the internal pressurized fluid. By means of the weight functions analytical technique, the leakage pressure can be calculated. The proposed model was then successfully validated by means of both numerical simulations and full scale and small scale experimental tests. The proposed analytical model can be used to compare different flange geometries and then it is a useful design tool.

Parole ChiAve. Tenuta. Flangia bullonata. Meccanica della frattura. Fessura parzialmente aperta. 


\section{INTRODUZIONE}

$\mathrm{I}$ compressori centrifughi di elevate dimensioni non prevedono l'utilizzo di guarnizioni di tenuta in materiale deformabile, che invece vengono comunemente usati per flangie di minore dimensioni. La tenuta fra le due metà della cassa del compressore è garantita dal contatto diretto (definito come "metal-to-metal") fra le due superfici della flangia, Fig.1. Tali superfici sono premute da una distribuzione di bulloni opportunamente preserrati. Nonostante non esista una guarnizione viene comunque applicato un opportuno sigillante immediatamente prima di portare a contatto le superfici, al fine di migliorare la prestazione di tenuta della flangia, principalmente per riempire gli inevitabili solchi di rugosità nonostante la prescritta elevata finitura superficiale. L'utilizzo del sigillante è di fatto la norma, nonostante la flangia venga definita come "metal-to-metal".

In letteratura sono reperibili studi recenti sulle condizioni di perdita di flange senza guarnizione, tuttavia non esiste un modello di tenuta che descriva il fenomeno in funzione dei parametri macroscopici geometrici. I principali risultati riportati in letteratura sono:

$\checkmark$ la planarità della superficie ha un ruolo significativo, la tolleranza di planarità deve essere molto stretta al fine di evitare perdite locali di contatto che producono un canale di perdita preferenziale [1];

$\checkmark$ in modo analogo, anche se ad un livello di scala differente, la rugosità deve essere minima per sfavorire perdite dovute ad un contatto non completo fra le superfici della flangia [2];

$\checkmark$ l'orientamento dei solchi di rugosità deve essere non allineato con l'eventuale verso del flusso di perdita, quindi possibilmente ortogonale ad esso [3];

$\checkmark$ l'irregolarità della superficie e la rugosità vengono in buona parte compensate con l'introduzione del sigillante (tipicamente siliconico) $[4,5]$.

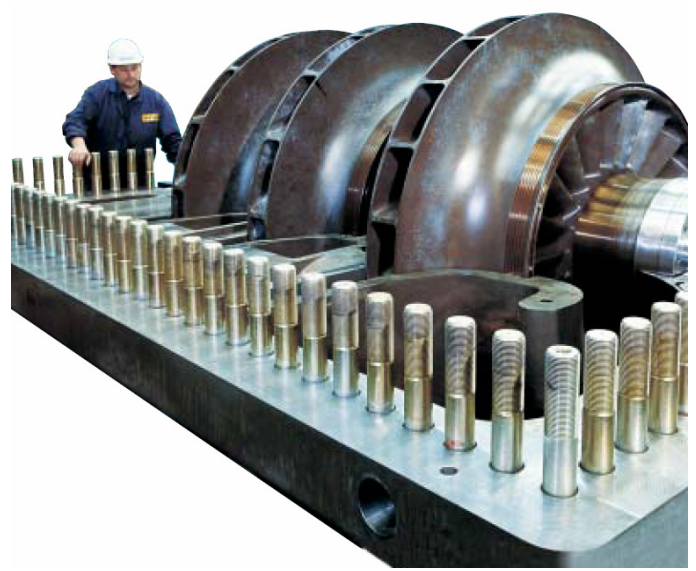

Figura 1: Tipiche dimensioni di un compressore centrifugo e relativa flangia bullonata di tenuta.

Molti studi presentano analisi agli Elementi Finiti (EF), utilizzando elementi di contatto (che comportano analisi di tipo non lineare) per determinare la distribuzione delle pressioni di contatto fra le due superfici della flangia [6-18], spesso offrendo soltanto analisi di carattere comparativo fra diverse configurazioni. Alcuni studi dimostrano l'effettiva importanza del sigillante [11]. La condizione di perdita è solitamente associata alla perdita di pressione di contatto fra le flange accoppiate [6-10] oppure al verificarsi di una tensione di trazione sufficiente a provocare il distacco fra il sigillante ed una delle due superfici della flangia [6]. L'effettivo valore del preserraggio imposto al bullone è ampiamente accettato come una delle principali cause di non affidabilità della flangia bullonata in termini di tenuta. Infine, alcuni studi propongono analisi su come ottimizzare la sequenza di serraggio per garantire un preserraggio dei bulloni il più possibile uniforme [14-21].

Il presente lavoro ha come obbiettivo quello di proporre un modello semplice ed efficace, in grado di determinare la condizione di perdita della flangia senza guarnizione, descrivendo la (parziale) separazione delle superfici della flangia con concetti di meccanica della frattura, ossia modellando l'interfaccia di separazione come una vera e propria fessura. Questo approccio permette di ottenere un modello analitico più veloce rispetto ad un calcolo agli elementi finiti, che quindi si presta ad un'analisi preliminare e di prima ottimizzazione dei parametri macroscopici della geometria della connessione. 
La geometria della connessione è rappresentata in Fig.2(a). I parametri geometrici principali sono: $D_{\mathrm{V}}$ diametro interno della cassa, $t_{\mathrm{V}}$ spessore della parete della cassa, $Z$ posizione dell'asse del bullone (o del prigioniero) rispetto alla superficie interna della cassa, $d_{\mathrm{H}}$ diametro del foro del bullone, $P_{\mathrm{B}}$ passo della fila di bulloni in direzione assiale, $H$ altezza di ciascuna delle superfici della flangia, $W$ larghezza della flangia, ed infine $L=Z-d_{\mathrm{H}} / 2$ è la "distanza di perdita" ossia l'estensione della separazione fra le due superfici della flangia che porta in comunicazione il volume interno, contenente fluido in pressione, con il foro del bullone che quindi è aperto verso l'esterno (in quanto il collegamento filettato non garantisce nessun tipo di tenuta). In altre parole, se la lunghezza di separazione $L_{0}$ fra le due superfici della flangia è inferiore a $L$ non si ha perdita, mentre si ha immediatamente perdita quando la lunghezza di separazione $L_{0}$ raggiunge la lunghezza $L$, Fig.2(b).

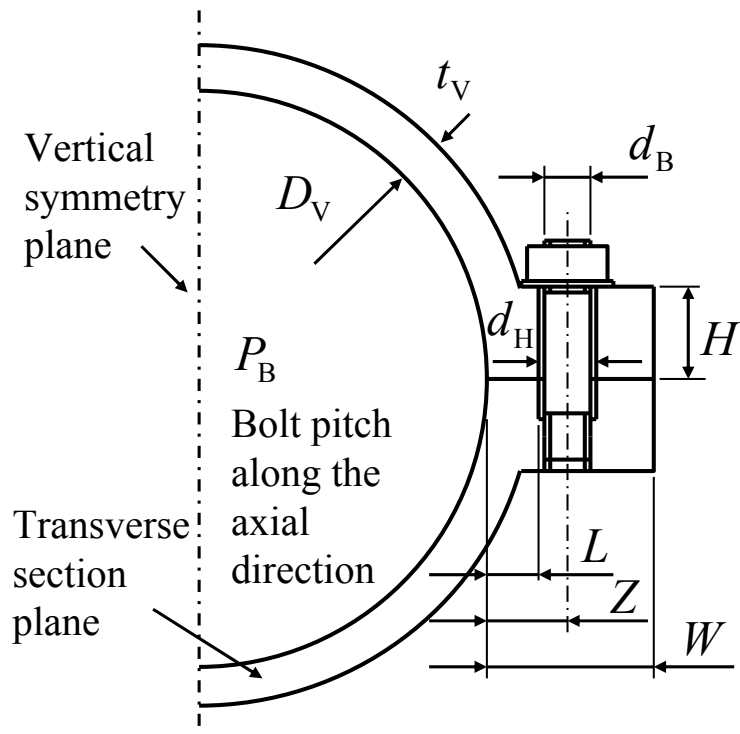

(a)

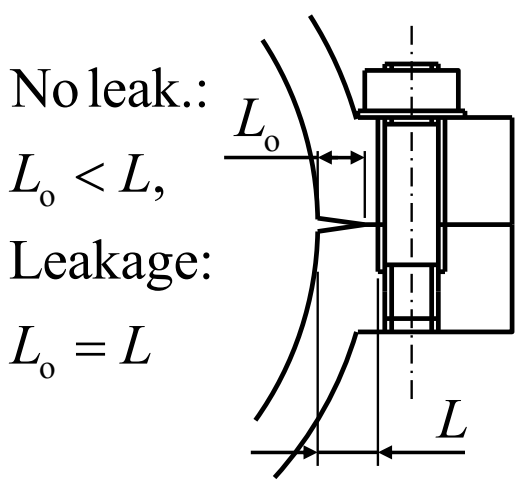

(b)

Figura 2: (a) Dimensioni principali della geometria della flangia. (b) Condizione di perdita.

La condizione di perdita assunta nel presente modello prevede che le superfici della flangia siano inizialmente perfettamente piane, e che la loro deformazione sia dovuta soltanto alla deformazione elastica, mentre invece le superfici posso presentare degli errori di forma (ad esempio dovuti al rilassamento di tensioni residue) e/o difetti locali come la rugosità oppure solchi o graffi nonostante l'applicazione del sigillante.

Un analisi EF di contatto ha permesso di verificare la pressione interna prevista dal modello che porta il fronte di separazione in corrispondenza del foro del bullone. Tuttavia, un'analisi numerica non può permettere di verificare la qualità dell'assunzione di perfetta planarità delle superfici che invece richiede una validazione sperimentale. Tale validazione è stata ottenuta (ed è presentata nel lavoro) mediante prove sia in piena scala sia in scala ridotta.

\section{MODELlo ANALITICO}

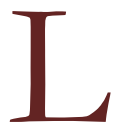

a porzione di distacco fra le superfici della flangia può essere interpretata come una vera e propria fessura. Le due piastre della flangia sono semplicemente appoggiate, tuttavia la zona in cui il contatto rimane chiuso è equivalente, in termini di stato di tensione, ad un'unica porzione di materiale senza soluzione di continuità, in quanto non si hanno slittamenti significativi.

Essendo le due piastre a contatto non è possibile avere uno stato di tensione positiva (trazione) fra le due superfici. Anche la presenza del sigillante non garantisce uno stato di trazione significativo, ma soltanto l'opportunità di riempire i solchi della rugosità. Come ben noto dalla meccanica della frattura, lo stato di tensione in corrispondenza dell'apice della fessura è definito dal fattore di amplificazione delle tensioni $K$. Il fattore di amplificazione (primo modo di apertura) non può mai essere negativo dal momento che questa condizione implica il contatto fra i lembi della fessura. D'altro canto la 
condizione di semplice contatto fra le superfici della piastra non permette un fattore di amplificazione positivo, dato che in tal caso le tensioni all'apice della fessura dovrebbero essere positive, addirittura singolari, per cui molto elevate in un introno dell'apice stesso. Quindi, il fattore di amplificazione è necessariamente nullo in corrispondenza del fronte di separazione. La condizione di perdita pertanto può essere espressa in termini di meccanica della frattura: fronte di separazione esteso fino al foro del bullone $L_{0}=L$ e fattore di intensificazione nullo $K=0$, Fig.3(a).

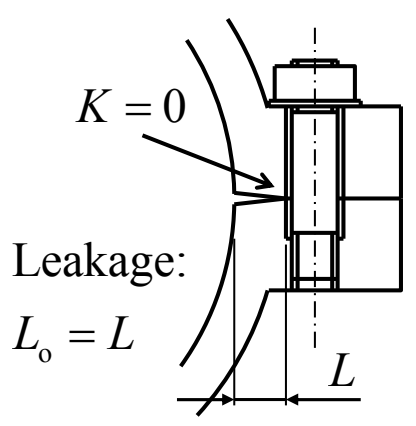

(a)

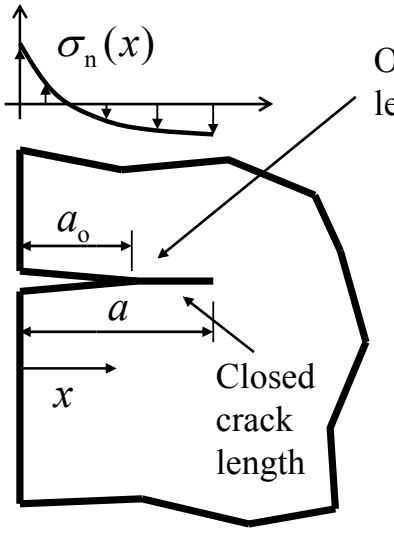

(b) length

Open crack

$$
\begin{aligned}
& K\left(a_{\mathrm{o}}\right)=0 \\
& K\left(a_{\mathrm{o}}\right)=\int_{0}^{a_{0}} \sigma_{\mathrm{n}}(x) h\left(x, a_{0}\right) \mathrm{d} x \\
& \int_{0}^{a_{0}} \sigma_{\mathrm{n}}(x) h\left(x, a_{0}\right) \mathrm{d} x=0
\end{aligned}
$$

Figura 3: (a) Condizione di perdita espressa in termini di meccanica della frattura.

(b) Integrazione della weight function, caso di una fessura parzialmente aperta.

La tecnica delle "Weight Functions" (WF) permette di esprime il fattore di intensificazione delle tensioni di una fessura come integrale esteso su tutta la lunghezza della fessura (in uno schema piano, altrimenti su tutta la superficie della fessura, in uno schema tridimensionale) della tensione nominale moltiplicata per una funzione "kernel" che è appunto la WF [2226].

La condizione di perdita può quindi essere scritta nel seguente modo, Eq.(1):

$$
K=\int_{0}^{L} \sigma_{\mathrm{n}}(x) h(x, L) \mathrm{d} x=0
$$

in cui la $\sigma_{\mathrm{n}}(x)$ è la distribuzione di tensione "nominale", mentre la $h(x, L)$ è la WF.

Come ben noto, la tensione nominale è quella distribuzione di tensione che si avrebbe in corrispondenza della linea della fessura (in uno schema piano) se la fessura non ci fosse, ossia se il materiale fosse continuo. È importante sottolineare che la WF $h(x, L)$ è soltanto funzione della geometria e non della tensione nominale. Tuttavia, la WF $h(x, L)$ e la tensione nominale $\sigma_{\mathrm{n}}(x)$ non possono essere espresse in forma chiusa per questa particolare geometria, quindi sono necessarie delle semplificazioni per ottenere una buona approssimazione della tensione nominale e della WF.

Nel presente problema della flangia bullonata la distribuzione di tensione nominale è la sovrapposizione della distribuzione di tensione dovuta al serraggio del bullone (che ovviamente produce tensioni di compressione, ovvero negative) e la distribuzione di tensione dovuta alla pressione interna alla cassa, che tende a distaccare le due piastre della flangia (tensioni di trazione, positive). La WF non è uniforme ma è comunque sempre positiva, per cui le tensioni nominali di trazione tendono a produrre $K$ positivo, mentre le tensioni di compressione dovute al preserraggio del bullone producono un contributo negativo, e quindi benefico ai fini della tenuta.

Di seguito si riportano le approssimazioni introdotte:

$\checkmark$ schema di calcolo piano, per cui la ripetizione dei fori viene schematizzata come un'unica cava continua di area equivalente, in modo da garantire, la stessa area di contatto, Fig.4(a), questa assunzione è incentivata anche dal fatto che il passo dei bulloni $P_{\mathrm{B}}$ è più piccolo possibile, in modo da favorire l'azione di serraggio stessa;

$\checkmark$ in virtù di questa assunzione, si fa riferimento ad uno schema piano trascurando la ripetizione ciclica dei fori, per cui la WF di integrazione è relativa ad uno schema di fessura nel piano;

$\checkmark$ per semplicità si trascurano gli effetti di bordo e si utilizza la WF di una fessura (di lunghezza finita) in un semipiano, di cui sono noti gli integrali per le più semplici distribuzioni di tensione nominale; 
$\checkmark$ si assume la distribuzione lineare per la tensione nominale dovuta alla pressione interna, e si determina tale distribuzione imponendo l'equivalenza (risultante e momento risultante) con la forza di trazione, per unità di profondità, attraverso la parete della cassa, Fig.4(b);

$\checkmark$ si assume la distribuzione della tensione nominale di compressione, dovuta al preserraggio dei bulloni, a tronco di piramide, rifacendosi al comune schema usuale nei testi di costruzione di macchine [27], avendo precedentemente assunto uno schema piano, la distribuzione a tronco di cono non è possibile, la relativa vicinanza dei bulloni $\left(P_{\mathrm{B}}\right.$ più piccolo possibile) ha suggerito la assunzione a tronco di piramide;

$\checkmark$ essendo la flangia relativamente stretta rispetto alla larghezza della distribuzione delle tensioni di compressione dovute al preserraggio dei bulloni, una porzione di tale distribuzione cade fuori dalla larghezza della flangia, al fine di garantire l'equivalenza è necessario sovrapporre una distribuzione equilibrante, assunta anch'essa lineare, equivalente alla distribuzione che cade fuori dalla larghezza della flangia, Fig.4(c).

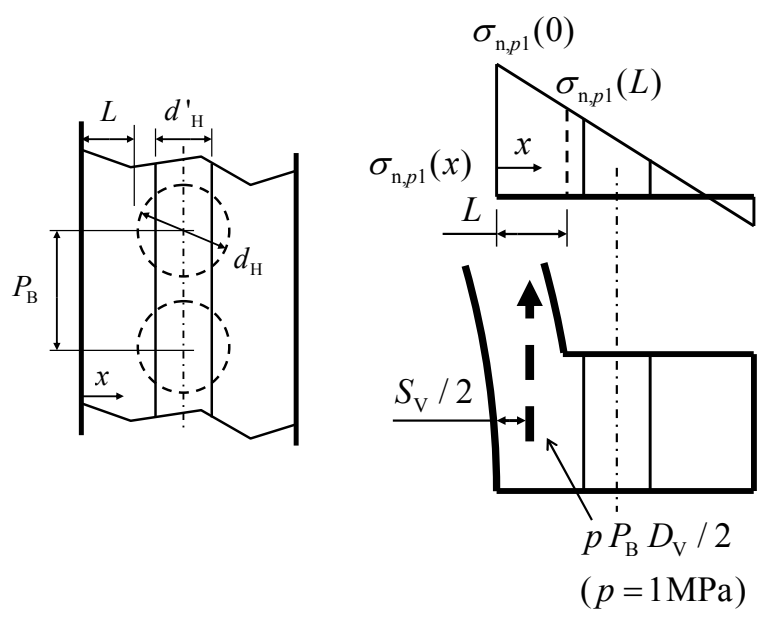

(a) (b)

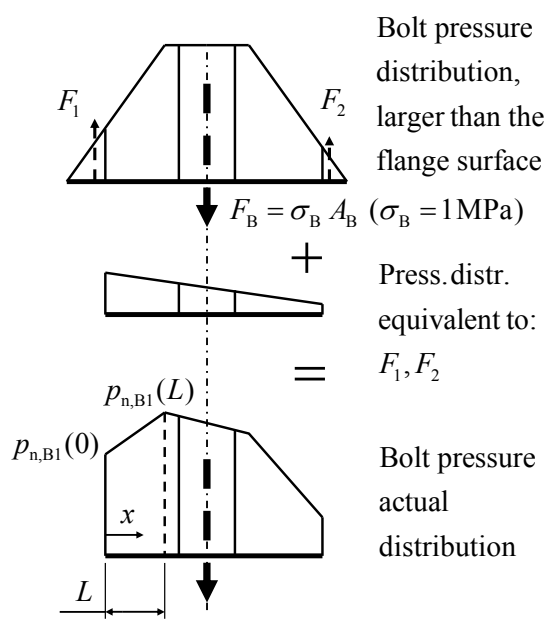

(c)

Figura 4: (a) Approssimazione geometrica della fila di bulloni come un'unica cava continua di area equivalente.

(b) Assunzione di distribuzione lineare delle tensioni nominali di trazione dovute alla pressione interna.

(c) Assunzione di distribuzione delle tensioni nominali di compressione dovute al preserraggio dei bulloni, re-distribuzione delle tensioni fuori dalla larghezza della flangia.

Le distribuzioni nominali (pressione interna e preserraggio dei bulloni) possono essere ottenute imponendo rispettivamente pressione interna alla cassa unitaria, e tensione di preserraggio del bullone anch'essa unitaria e successivamente moltiplicando per l'effettiva pressione interna e l'effettiva tensione di preserraggio. La distribuzione di tensione nominale può quindi essere espressa mediante la seguente combinazione lineare:

$$
\sigma_{\mathrm{n}}(x)=p \sigma_{\mathrm{n}, p 1}(x)-\sigma_{\mathrm{B}} p_{\mathrm{n}, \mathrm{B} 1}(x)
$$

Da notare che la tensione nominale prodotta dalla pressione interna è positiva (trazione), mentre la tensione nominale prodotta dal preserraggio e negativa (pressione).

Avendo assunto distribuzioni lineari delle componenti della tensione nominale, l'integrazione della WF si riduce alla combinazione lineare dell'integrazione di una distribuzione uniforme e di una variabile linearmente, Fig.5.

Sostituendo l'Eq.2 nell'Eq.1, e avendo i risultati delle integrazioni, riportati nella Fig.5, è possibile ottenere il valore di pressione di perdita:

$$
p_{\mathrm{L}}=\sigma_{\mathrm{B}} \frac{p_{\mathrm{n}, \mathrm{B} 1}(0)+1.55 p_{\mathrm{n}, \mathrm{B} 1}(L)}{\sigma_{\mathrm{n}, p 1}(0)+1.55 \sigma_{\mathrm{n}, p 1}(L)}
$$

Nell'Eq.3, i termini $p_{\mathrm{n}, \mathrm{B} 1}(0), p_{\mathrm{n}, \mathrm{B} 1}(L)$ e $\sigma_{\mathrm{n}, p 1}(0), \sigma_{\mathrm{n}, p 1}(L)$ sono $\mathrm{i}$ valori di tensione nominale per $x=0$ e $x=L$, rispettivamente, ossia alla posizione interna della cassa e alla distanza di perdita.

Tuttavia, quando una porzione delle superfici della flangia perde contatto, inevitabilmente penetra del fluido in pressione, che tende ad incentivare la separazione fra le due superfici. Al fine di considerare tale effetto, seguendo l'approccio proposto, è sufficiente aggiungere un termine di trazione alla distribuzione di tensione nominale, pari al valore della 
pressione interna stessa. Dato il significato dei termini $\sigma_{\mathrm{n}, p 1}(0), \sigma_{\mathrm{n}, p 1}(L)$ e grazie alla linearità delle integrazioni della WF, è quindi necessario aggiungere ad entrambi un termine unitario. L'Eq.4 costituisce il modello analitico definitivo per determinare la pressione di perdita:

$$
p_{\mathrm{L}}=\sigma_{\mathrm{B}} \frac{p_{\mathrm{n}, \mathrm{B} 1}(0)+1.55 p_{\mathrm{n}, \mathrm{B} 1}(L)}{\left(\sigma_{\mathrm{n}, p 1}(0)+1\right)+1.55\left(\sigma_{\mathrm{n}, p 1}(L)+1\right)}
$$

Come descritto in precedenza le tensioni nominali unitarie possono essere dedotte sulla base di considerazioni di equilibrio, anche se approssimate. Pertanto non è necessaria alcuna simulazione numerica per ottenere i termini che compaiono nell'Eq.3. Tuttavia, il modello EF successivamente riportato è stato sviluppato al fine di verificare i risultati del modello analitico.

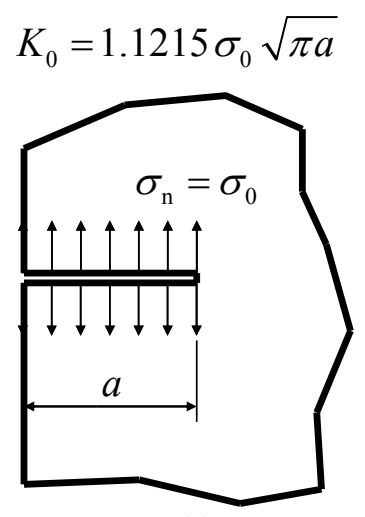

(a)

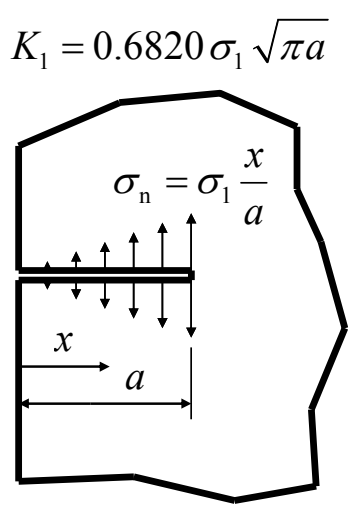

(b)

Figura 5: (a) Integrazione della WF con distribuzione di tensione nominale uniforme.

(b) Integrazione della WF con distribuzione di tensione nominale variabile linearmente.

\section{MODELLO ELEMENTI FINITI}

a porzione di flangia modellata agli elementi finiti è rappresentata in Fig.6(a). L'analisi si limita alla porzione rettilinea delle flange ed inoltre si sfruttano le due simmetrie dovute alla ripetizione geometrica dei bulloni, Fig.6(b).

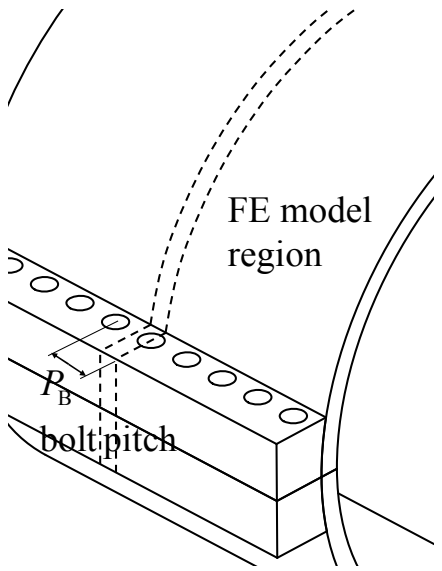

(a)

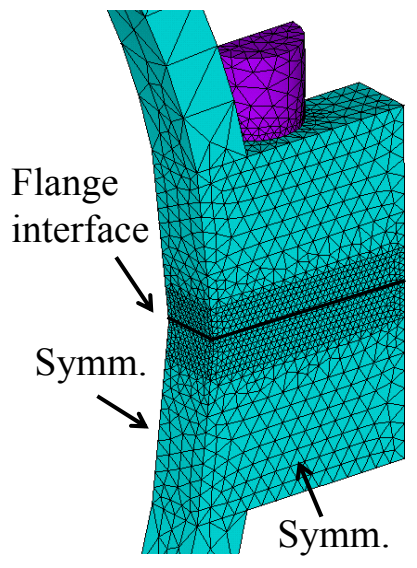

(b)

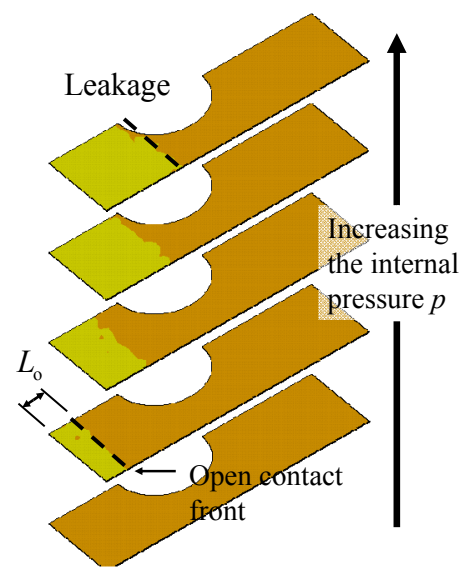

(c)

Figura 6: (a) Porzione di flangia modellata. (b) Modello EF, utilizzo delle simmetrie.

(c) Distacco degli elementi di contatto e condizione di perdita

In Fig.6(c) si mostra l'evoluzione del fronte di apertura all'aumentare della pressione interna alla cassa, fino alla condizione di perdita, ossia quando il fronte di distacco raggiunge il punto più interno del perimetro del foro del bullone. Il valore 
della pressione di perdita prevista dal modello $\mathrm{EF}, p_{\mathrm{L}, \mathrm{FE}}$, si determina semplicemente osservando la posizione del fronte di apertura, per piccoli incrementi della pressione interna. È importante sottolineare che, nonostante la non linearità di contatto, la pressione di perdita prevista dal modello EF, è legata linearmente al preserraggio del bullone, dato che si impone una specifica posizione del fronte di apertura. Questo risultato è in accordo con l'evidente linearità, prevista dal modello analitico, fra pressione di perdita $p_{\mathrm{L}}$ e il preserraggio del bullone.

In Fig.7 si mostra l'ottima correlazione fra la pressione di perdita prevista dal modello analitico rispetto alla pressione di perdita prevista dal modello EF, per 12 diverse configurazioni di casse. È quindi evidente che le approssimazioni introdotte non hanno prodotto errori significativi. Tuttavia, come già accennato nell'Introduzione, il modello EF rappresenta una validazione per il modello analitico in termini di previsione della posizione del fronte di apertura, mentre la modalità di perdita assunta, ossia il fluido che fuoriesce dalla cassa soltanto se il fronte di separazione fra le superfici delle flange raggiunge il bordo del foro del bullone, necessita di una conferma sperimentale.

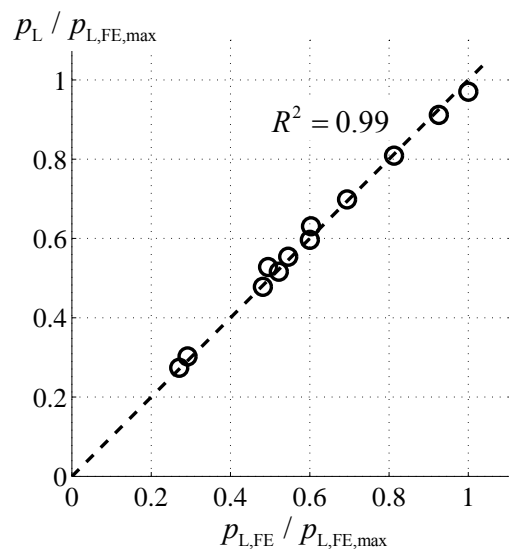

Figura 7: Correlazione fra la pressione di perdita prevista con modello EF e pressione di perdita prevista con modello analitico basato sulla meccanica della frattura.

\section{VALIDAZIONE SPERIMENTALE}

\section{Prove in piena scala}

T a Fig.8 riporta le prove di pressurizzazione in piena scala per le stesse 12 configurazioni precedentemente investigate con il modello EF. Ciascuna tipologia di cassa è stata testata prima di essere messa in esercizio, mediante prove di pressurizzazione. Questo tipo di prova prevede di introdurre del liquido (anche se si tratta di compressori per gas) raggiungendo un certo valore di pressione, maggiorato rispetto al valore di esercizio, e di verificare l'eventualità della perdita.

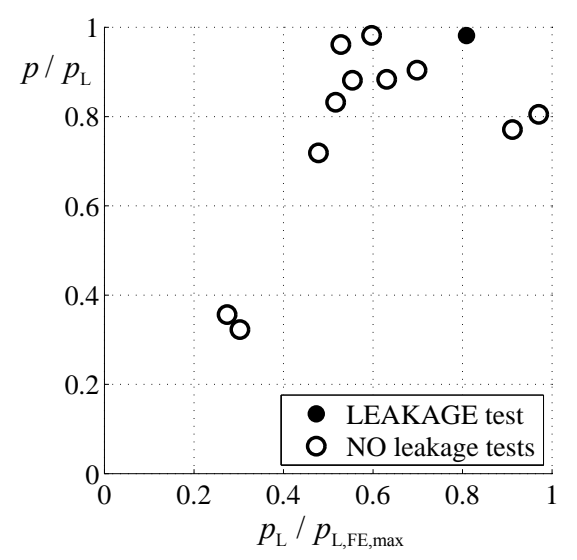

Figura 8: Prove in piena scala di pressurizzazione. Corretta previsione, mediante il modello analitico, del singolo caso di perdita. 
Soltanto per un caso è stato possibile portare la pressione interna ad un valore più elevato di quello previsto dalla prova di pressurizzazione, fino a raggiungere la perdita, Fig.8. Per tale prova, è stato opportunamente estensimetrato un bullone della flangia per conoscere con elevata confidenza l'effettivo preserraggio, mentre negli altri casi il precarico del bullone è stato soltanto stimato sulla base del valore imposto mediante il tensionatore idraulico. Da notare che nell'unico caso di perdita la previsione del modello è stata accurata, in quanto il rapporto $p / p_{\mathrm{L}}$ è risultato molto prossimo all'unità. Nelle altre configurazioni non è stata raggiunta la pressione di perdita, per necessità di servizio e quindi il margine di previsione del modello è rimasto incerto.

\section{Prove in scala ridotta}

Si è ritenuto opportuno eseguire ulteriori prove, in scala ridotta, per la validazione del modello analitico, in modo da monitorare con accuratezza il preserraggio dei bulloni e poter raggiungere la condizione di perdita senza particolari restrizioni. La Fig.9(a) mostra una vite estensimetrata, il relativo schema per l'acquisizione del segnale, in modo da misurare solo la trazione ed eliminare l'effetto di flessione e temperatura, mentre la Fig.9(b) mostra l'attrezzatura di prova in scala.
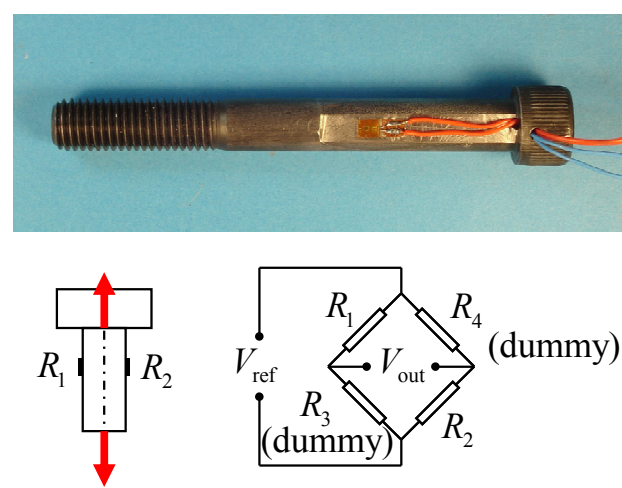

(a)

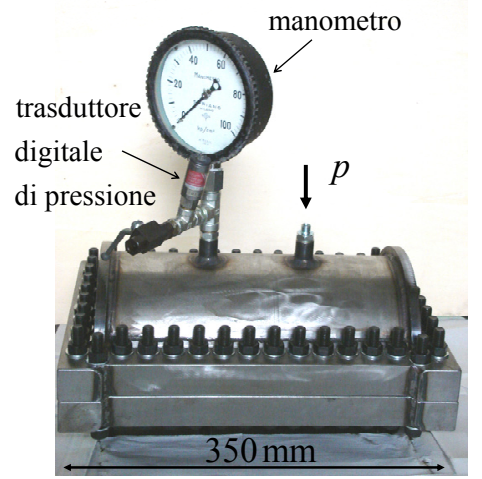

(b)

Figura 9: (a) Vite estensimetrata, l'utilizzo di due estensimetri permette di eliminare eventuale flessione ed effetto di temperatura, oltre ad ottenere sensibilità del segnale doppia. (b) Attrezzatura sperimentale per riprodurre in scala ridotta una cassa flangiata con bullonatura di tenuta.

I bulloni estensimetrati sono stati applicati nella zona centrale della flangia bullonata, ed è stato eseguito un serraggio controllato. Gli altri bulloni sono stati serrati con un precarico molto maggiore anche se non controllato. In questo modo la perdita è stata condizionata a manifestarsi in corrispondenza dei bulloni estensimetrati. Per ciascuna prova sono stati applicati incrementi di pressione fino al verificarsi della perdita, messa in evidenza dal liquido colorato introdotto, Fig.10.

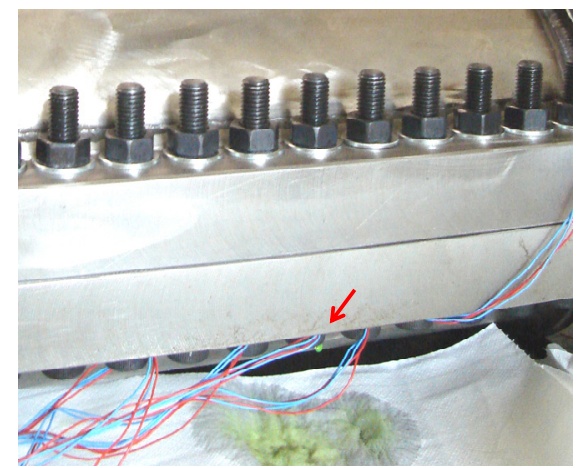

Figura 10: Prove in scala ridotta con liquido in pressione (acqua colorata). Evidenza di perdita

Superata la pressione di perdita, si manifesta il gocciolamento continuo con una certa frequenza. Ovviamente la frequenza di gocciolamento è funzione crescente della pressione interna del fluido. La portata di perdita è stata valutata misurando la massa di una singola goccia, misurando l'intervallo di tempo fra il manifestarsi di una goccia e la successiva e quindi dividendo massa per tempo. Riportando su un grafico la portata di perdita in funzione della pressione interna, è intuitivo definire come la pressione (sperimentale) di perdita il valore di intercetta di un andamento lineare approssimante delle singole misurazioni di portata di perdita, Fig.11. 


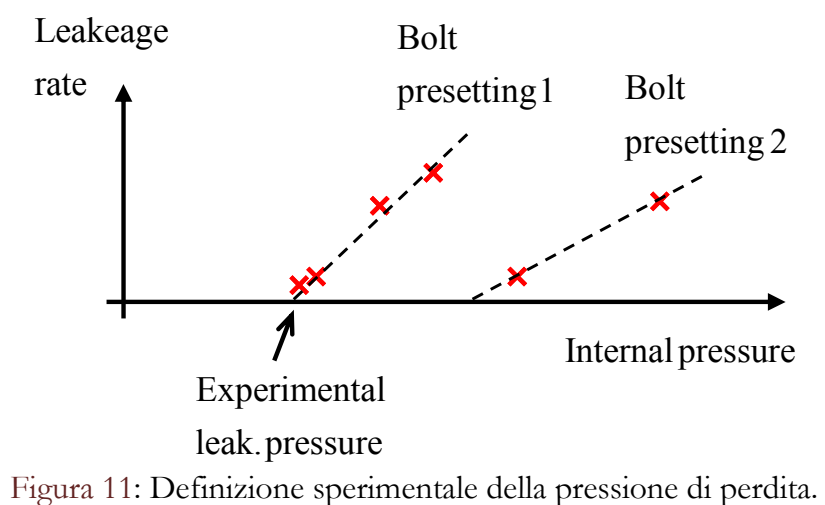

I risultati delle prove in scala ridotta sono riportati in Tab.1. L'errore percentuale di previsione della pressione di perdita non supera il $7 \%$.

\begin{tabular}{|c|c|c|c|c|}
\hline Prova & $\begin{array}{c}\text { Preserraggio } \\
\text { bulloni } \\
{[\mathrm{kN}]}\end{array}$ & $\begin{array}{c}\text { Pressione di perdita } \\
\text { sperimentale } \\
\text { [bar ] }\end{array}$ & $\begin{array}{l}\text { Pressione di perdita } \\
\text { prevista dal modello } \\
\text { [ bar ] }\end{array}$ & $\begin{array}{c}\text { Errore } \\
\text { percentuale }\end{array}$ \\
\hline 1 & 30.7 & 63 & 59 & $6 \%$ \\
\hline 2 & 20.2 & 41 & 41 & $<1 \%$ \\
\hline 3 & 20.1 & 43 & 41 & $6 \%$ \\
\hline 4 & 20.4 & 41 & 41 & $<1 \%$ \\
\hline 5 & 30.5 & 57 & 61 & $7 \%$ \\
\hline
\end{tabular}

Tabella 1: Risultati delle prove in scala ridotta.

\section{ANALISI DI SENSIBILITÀ AI PARAMETRI GEOMETRICI}

L

a disponibilità di un modello analitico semplice (e validato) in grado di valutare la pressione di perdita, rappresenta uno strumento di progetto molto utile, soprattutto in una prima fase di definizione dei parametri macroscopici della flangia. È stata quindi eseguita un'analisi di sensibilità al variare di un parametro tenendo costante gli altri ed ottenendo i seguenti risultati:

$\checkmark$ la pressione di perdita $p_{\mathrm{L}}$ è lineare con il preserraggio dei bulloni, risultato ovvio considerando l'Eq.3, per cui è buona norma scegliere bulloni di classe elevata in modo da poter sfruttare al meglio il preserraggio, al fine di aumentare $\sigma_{\mathrm{B}}$;

$\checkmark$ la pressione di perdita diminuisce all'aumentare del passo dei bulloni $P_{\mathrm{B}}$, Fig.12(a), in quanto l'azione media di preserraggio si riduce, oltretutto si tende a produrre una disuniformità della pressione di contatto in direzione assiale (non prevista dal modello), rischiando di avere una locale riduzione di pressione di contatto fra le superfici della flangia, in definitiva è buona norma ridurre il passo assiale dei bulloni al minimo tenendo conto degli ingombri;

$\checkmark$ la pressione di perdita aumenta all'aumentare della larghezza della flangia $W$, Fig.12(b), in modo non molto sensibile, fino ad un livello di saturazione, oltre al quale la pressione di perdita rimane costante nonostante un ulteriore aumento della larghezza della flangia;

$\checkmark$ la pressione di perdita è pressoché insensibile all'altezza della flangia $H$, Fig.12(c), qualora sia sufficientemente più grande della dimensione del bullone;

$\checkmark$ la pressione di perdita diminuisce all'aumentare della posizione dell'asse del bullone $Z$, ovvero la distanza dalla superficie interna, Fig.12(d), in quanto l'azione di preserraggio risulta più remota rispetto alla zona della flangia interessata dalla perdita.

La possibilità di avere maggiore pressione di perdita all'aumentare del preserraggio del bullone, apparentemente, potrebbe indurre a pensare che sia utile introdurre un diametro maggiore del bullone, in modo quindi da avere maggiore preserraggio. Tuttavia è bene tenere presente gli ingombri, mostrati in Fig.2(a). Aumentare il diametro del bullone provoca un aumento del passo e un aumento della distanza dalla superficie interna, e quindi un effetto negativo sulla pressione di perdita. D’altro canto un bullone di diametro piccolo produrrebbe una ridotta (in modulo) pressione di serraggio. 
Evidentemente, esiste un compromesso della dimensione di diametro del bullone, che massimizza la pressione di perdita. La disponibilità di un modello analitico, permette di trovare tale compromesso con un'analisi parametrica comparativa.

\section{CONCLUSIONI}

$\mathrm{I}$

1 presente lavoro propone un modello di tenuta dedotto sulla base della meccanica della frattura. La condizione di perdita è la parziale separazione delle superfici della flangia fino al raggiungimento del foro del bullone. La separazione può essere vista come una fessura parzialmente aperta, pertanto il fattore di intensificazione della fessura costituita dalle flange in contatto è necessariamente nullo. L'utilizzo delle "weight functions" ha permesso di descrivere tale condizione in funzione delle tensioni nominali, ossia delle tensioni che si avrebbero se la flangia fosse un unico componente. Al fine di ottenere un modello analitico facilmente risolvibile sono state introdotte delle semplificazioni, che tuttavia a posteriori si sono dimostrate lecite, in quanto le validazioni del modello (numerica e sperimentali) hanno dato esito positivo. Il modello analitico di tenuta proposto, non è in grado di valutare l'effetto di aspetti di dettaglio quali: lo stato della superficie (tolleranza di planarità, rugosità, presenza del sigillante), oppure la sequenza di serraggio dei bulloni che può generare disuniformità di preserraggio, oppure la presenza di un gas in pressione piuttosto che un liquido. Tuttavia, il presente modello permette di eseguire un'analisi comparativa di sensibilità ai principali parametri geometrici della flangia quali: passo assiale e distanza dalla superficie interna dell'asse dei bulloni, altezza e larghezza della flangia, offrendo quindi un utile strumento di progetto e di ottimizzazione.

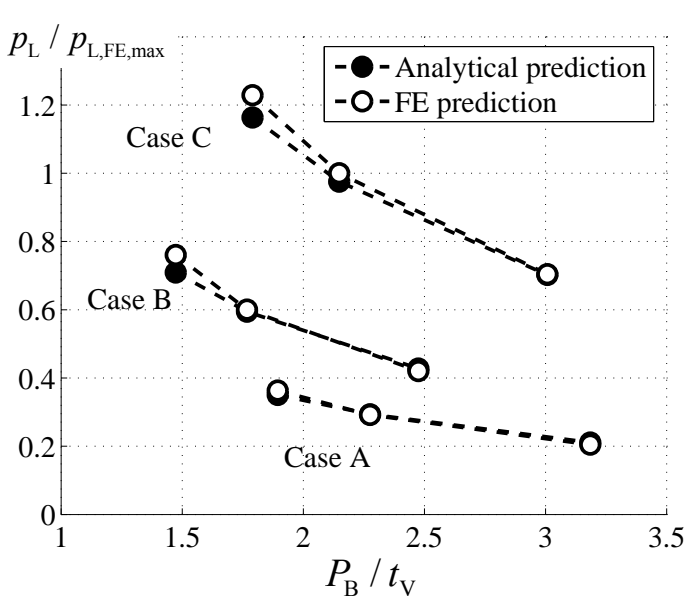

(a)

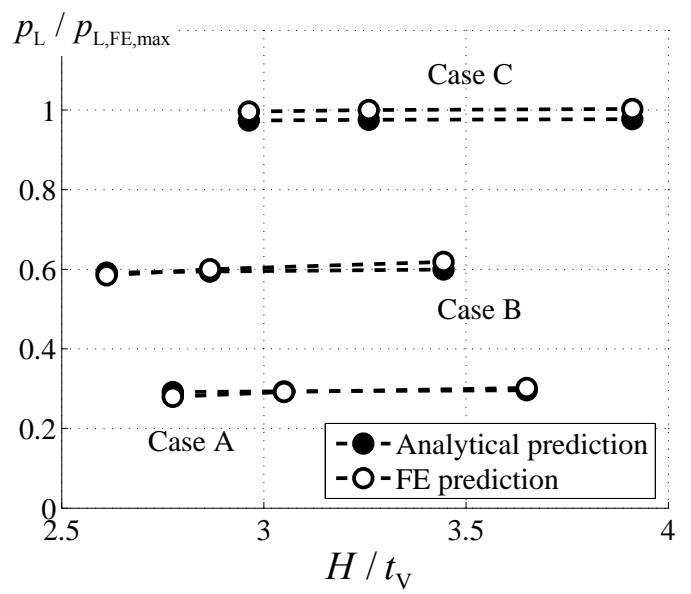

(c)

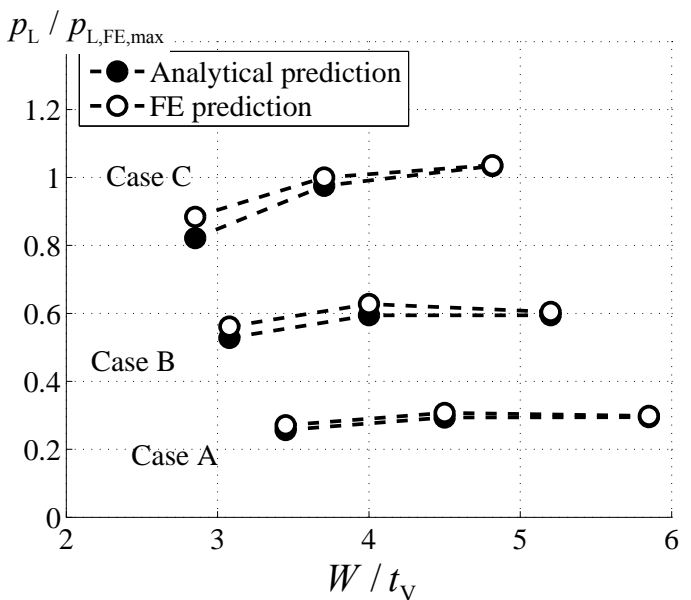

(b)

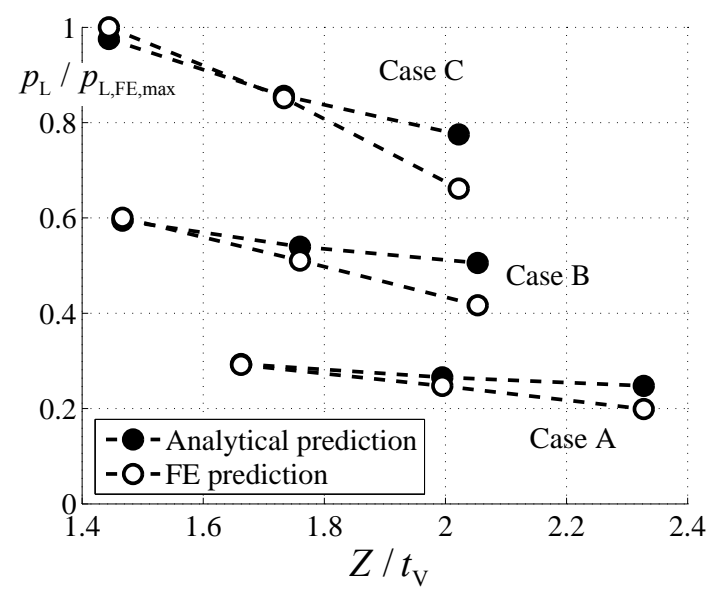

(d)

Figura 12. Sensibilità della pressione di contatto ai principali parametri geometrici: (a) passo assiale dei bulloni, (b) larghezza della superficie di contatto della flangia, (c) altezza della flangia, (d) posizione dell'asse del bullone. 


\section{BIBLIOGRAFIA}

[1] D.K. Nash, M. Abid, in: Proceedings of the Institution of Mechanical Engineers, Part E: Journal of Process Mechanical Engineering, 218 (4) (2004) 205.

[2] T. Nakamura, K. Funabashi, Lubrication Science, 4 (1) (1991) 13.

[3] J. Arghavani, M. Derenne, L. Marchand, The International Journal of Advanced Manufacturing Technology, 21 (1011) (2003) 713 .

[4] G. Murtagian, V. Fanelli, J. Villasante, D. Johnson, H. Ernst, Journal of Tribology, 126 (3) (2004) 591-596.

[5] F. Kirkemo, in: Proceedings of 2002 ASME Pressure Vessels and Piping Conference (PVP2002). Vancouver, BC, Canada, (2002) 1087.

[6] H. Kawamura, T. Sawa, M. Yoneno, Journal of Adhesion Science and Technology, 17 (8) (2003) 1109.

[7] M. Abid, D. Nash, International Journal of Pressure Vessels and Piping, 80 (12) (2003) 831.

[8] M. Abid, International Journal of Mechanics and Materials in Design, 2 (1-2) (2005) 35.

[9] M. Abid, International Journal of Pressure Vessels and Piping, 83 (6) (2006) 433.

[10] M. Abid, International Journal of Mechanics and Materials in Design, 2 (1-2) (2005) 129.

[11] E. Roos, H. Kockelmann, R. Hahn, International Journal of Pressure Vessels and Piping, 79 (1) (2002) 45.

[12] H. Estrada, I. Parsons, International Journal of Pressure Vessels and Piping, 76 (8) (1999) 543.

[13] T. Sawa, N. Ogata, Journal of Pressure Vessel Technology, 124 (4) (2002) 385.

[14] M. Abid, D. Nash, International Journal of Solids and Structures, 43 (14-15) (2006) 4616.

[15] T. Fukuoka, T. Takaki, Journal of Mechanical Design, 125 (4) (2003) 823.

[16] J. Mackerle, International Journal of Pressure Vessels and Piping, 82 (7) (2005) 571.

[17] J. Mackerle, International Journal of Pressure Vessels and Piping, 80 (4) (2003) 253.

[18] J. Mackerle, Finite Elements in Analysis and Design, 20 (3) (1995) 205.

[19] H. Tsuji, M. Nakano, in: Proceedings of 2002 ASME Pressure Vessels and Piping Conference (PVP2002). Vancouver, BC, Canada, (2002) 1094.

[20] T. Fukuoka, Journal of Pressure Vessel Technology, 127 (4) (2005) 402.

[21] T. Fukuoka, T. Takaki, Journal of Pressure Vessel Technology, 125 (4) (2003) 371.

[22] M. Beghini, L. Bertini, Engineering Fracture Mechanics, 54 (5) (1996) 667.

[23] M. Beghini, L. Bertini, V. Fontanari, Fatigue \& Fracture of Engineering Materials \& Structures, 28 (1-2) (2005) 31.

[24] M. Beghini, L. Bertini, V. Fontanari, International Journal of Fracture, 112 (1) (2001) 57.

[25] X.-R. Wu, A. Carlsson, Weight Functions and Stress Intensity Factor Solutions. Pergamon Press, Oxford (1991).

[26] T. Fett, D. Munz, Stress Intensity Factors and Weight Functions. Computational Mechanics, Billerica, MA (1997).

[27] J. Shigley, C. Mischke, R. Budynas. Mechanical Engineering Design, 7 ed. McGraw-Hill Science/Engineering/Math, (2003). 